Voix et Images

volxetimages

\title{
L'idéologie de la modernité ou le fantasme du nouveau
}

\section{Michel Coutu}

Volume 11, numéro 3 (33), printemps 1986

Yolande Villemaire

URI : https://id.erudit.org/iderudit/200584ar

DOI : https://doi.org/10.7202/200584ar

Aller au sommaire du numéro

Éditeur(s)

Université du Québec à Montréal

ISSN

0318-9201 (imprimé)

1705-933X (numérique)

Découvrir la revue

Citer cet article

Coutu, M. (1986). L'idéologie de la modernité ou le fantasme du nouveau. Voix et Images, 11(3), 507-520. https://doi.org/10.7202/200584ar d'utilisation que vous pouvez consulter en ligne.

https://apropos.erudit.org/fr/usagers/politique-dutilisation/ 


\title{
L'idéologie de la modernité ou le fantasme du nouveau ${ }^{1}$
}

\author{
par Michel Coutu, Université du Québec à Montréal
}

Ce texte propose une lecture des procédés discursifs circulant autour de «l'idée de modernité». À travers les procédés formels et discursifs du discours sur la modernité, il propose de voir comment l'idée de "faire différent" se constitue en idéologie du différent (et même du «différend" dans certains cas!).

Des contributions récentes ont mis à jour des approches et des acceptions très différenciées de la modernité (voir entre autre: Possibles, vol. 8 no 3). Si ces témoignages font état de conceptions divergentes, ils laissent cependant un vide profond quant à son procès: on a l'impression que le texte moderne s'articule comme par magie, qu'il fonctionne parce qu'il fonctionne, avec tout ce que ceci peut appeler de rituel avec ses initiés et ses formules incantatoires, autant prescriptives que proscriptives.

Notre objectif est donc d'étudier ce procès. Le champ d'étude se limitera aux chroniques se rapportant ou faisant appel à la notion de modernité parues dans le magazine Spirale de l'année de publication 1983, de même qu'aux actes du colloque sur la Nouvelle Écriture (NBJ no 90-91, 1982) ${ }^{2}$. Ce choix se justifie au regard des développements récents, entre autres les publications suivantes: Qui a peur de l'écrivain?, les Herbes Rouges nos 123-124, et Vouloir la fiction, $\odot$ La Modernité, NBJ no 141; on sent en effet dans ces textes (de haute polémique) un certain malaise à l'emploi du terme de modernité. Et, comme le terme lui-même s'est comme usé, saturé par un emploi quelque peu abusif, on dénote une tendance à retourner au concept d'origine, celui de la Nouvelle Écriture (N.E.), opérant ainsi un repli (que l'on pourrait qualifier de stratégique) vers l'origine, vers la tradition de rupture...

Le magazine Spirale est avant tout l'instance critique du texte moderne. Il est un lieu d'élection et de sélection, et des luttes s'y jouent, provoquant jusqu'à un certain point la parution des deux ouvrages ci-haut mentionnés. C'est précisément au cours de l'année étudiée que se sont démarquées des tendances: là où on parlait de modernité (consensuelle), on parle bientôt de modernités, de certaines modernités, de lieux dits modernes, etc. Autant de symptômes que la modernité ne va plus de soi (enfin, pas pour tout le monde!).

À partir des pratiques discursives des chroniques et des contributions au colloque sur la Nouvelle Écriture, nous avons prélevé les unités lexicales qui 
definissent et organisent le discours sur la modernité. L'emploi d'énoncés méliorés et péjorés indique que l'idée de modernité relève d'une conception "organique" du texte moderne, dans ce sens précis où l'on perçoit la modernité comme un Tout Synthétique (l'expression est de M. Angenot). Enfin, la modernité, comme tout système d'idée organisée et diffusée, n'échappe pas à l'emploi de présupposés en rapport à son objet, ceux-ci finissant par s'imposer, dans la pratique critique, comme poncifs esthétiques.

\section{La modernité comme idéologie}

...plus je me sens et me sais me rapprocher de ce temps où je serai tellement moderne que plus rien ne sera possible après et tout l'avant tout mon passé sera définitivement terminé. Je me serai alors dépassée moi-même.

Madeleine Gagnon, in

Possibles vol. 8, no 3.

En quoi le concept de modernité tiendrait-il de l'idéologique, et quel procès mettrait-il en cause s'il procède d'un tel système? Ou encore, peut-on situer le discours sur la modernité (qu'il faut distinguer du discours de la modernité) comme devant composer avec la compétence linguistique et idéologique?

Une fois posée la situation spécifique de l'avant-garde et de la circulation restreinte de ses productions, il semble bien que le discours critique sur la modernité, à travers le magazine Spirale du moins, pose la modernité comme seule voie d'écriture et de production d'œuvres esthétiques. C'est dans ce sens que la modernité agirait comme un système idéologique, se réclamant d'un Tout Synthétique; ce Tout Synthétique organise un système prescriptif en regard de la création, émettant ses poncifs esthétiques et ses mots d'ordre sur l'élaboration de "certaines modernités" (dans le sens de vouloir en contrôler l'usage).

Pour des raisons d'économie, nous aborderons l'idéologie de la modernité dans son sens le plus restreint, c'est-à-dire que nous nous attacherons plus à la circulation des termes qui y "gravitent " qu'aux différentes conceptions que s'en font les agents de divers lieux où elle se retrouve.

Pour l'articulation idéologique de la modernité, nous nous référons aux cinq traits par lesquels $\mathrm{O}$. Reboul ${ }^{3}$ définit une idéologie dans son rapport au langage. Il s'agit de 1- une pensée partisane, 2- une pensée collective, 3- une pensée dissimulatrice, 4- une pensée rationnelle, et 5- une pensée au service du pouvoir 4 . Il va sans dire que pour nous, «l'idéologie de la modernité» n'a de valeur que dans la mesure où elle est en relation avec une ou plusieurs autres idéologies. Autrement dit, il n'est nullement question de restreindre l'idéologie à son singulier: il n'y a d'idéologies que plurielles, dans les rapports qu'elles entretiennent avec les autres conceptions du monde que peuvent avoir divers agents du même groupe social ou d'un groupe social différent de la société dans laquelle ils vivent. 
En tant que pensée partisane, toute idéologie se situe dans un conflit d'idéologies s, et le magazine Spirale est au centre de ce conflit: il agit comme polarisateur des tiraillements idéologiques qui secouent la modernité. Ceux-ci se situent à plusieurs niveaux: entre ancien/moderne, entre une conception "pluraliste» de la modernité et une conception prescriptive (voir plus loin), entre forme/contenu, entre notions "périmées" (goût, valeurs, etc.) et concepts modernes (écriture, rupture, etc.); bref, entre les luttes séculaires de l'histoire littéraire.

En tant que pensée collective, une idéologie est une pensée anonyme, un discours sans auteurs, ce que tout le monde "croit" sans que personne ne le pense (ibid.). Tous les intervenants se réclamant de la modernité, celle-ci fonctionne à l'implicite et semble, pendant un certain temps, relever du consensus. L'absence de retour critique sur la modernité, jusqu'à ce qu'un "certain contenu» ne vienne remettre en question le concept, est symptomatique d'une "idée consensée", de ce que la modernité s'impose à tous pour tomber sous le sens: on "sent" la chose moderne plus qu'on ne la définit.

Une idéologie est dissimulatrice (à un niveau non conscient) dans ce qu'elle se donne toujours pour autre chose que ce qu'elle est (ibid). La modernité est dissimulatrice dans le sens où son discours tend à lire autrement ce qui interpelle (31.3), sans vraiment jeter les bases théoriques ou épistémologiques de cette «lecture moderne", sans jamais donner les sources de ce "savoir" où lecture, comme écriture, participent à l'effet moderne: le texte de la Nouvelle Écriture fonctionne à l'implicite (NBJ p. 167).

Or, et étrangement, rien n'est plus implicite lorsqu'on a affaire à un texte dit "ancien» (entendre procédés formels «désavoués» par la modernité). On appelle alors tout l'appareil théorique (narratologie, structuralisme, psychanalyse, féminisme, etc.) pour conclure, presque dans chaque cas que, même s'il y a "écriture", l'auteur étale son ignorance de la modernité (c'est-à-dire de ses procédés formels): des récits, donc, qui semblent avoir oublié qu'il n'est plus possible dorénavant de faire comme si la théorie sur le roman n'existait pas (33.15). La modernité est une pensée dissimulatrice aussi en ce qu'elle appelle des mythes contemporains sans retour critique sur cette nouvelle «mythologie moderne»: la Ville, l'Urbanité, et même le Pharaonisme(!), etc., participent tous à l'édifice moderne.

Une pensée rationnelle: ici se pose le problème de la validité de l'hypothèse. L'idée de modernité tend à échapper au rationalisme dans le sens qu'elle se présente toujours pour ce qu'elle n'est pas, qu'elle se donne pour insaisissable; décortiquant les états du désir d'écrire, il me reste toujours un résidu d'indicible, un inexplicable (NBJ p. 112); (la N.E.) ne vit que de sa propre absence de définition (NBJ p. 122).

Le rationnel agirait plutôt ici sous l'ordre du négatif: on ne rationalise que pour ce qui ne tient pas le pari de la modernité. L'organisation discursive des critiques apparaît alors comme un prétexte à la théorisation, le théorique devenant le seul référent (pour les textes que l'on dit peu ou pas modernes); à l'inverse, pour le discours sur la modernité, on évacue ce référent (le théo- 
rique) pour le faire jouer à l'implicite: je lis autrement car ce livre m'interpelle (31.3) dénote assez bien que "lire autrement", c'est faire passer le texte moderne sous un autre registre. Cet "Autre» reste immanquablement implicite et la critique (il serait plus juste de parler de "lecture") reste au niveau de l'intuitif.

Une pensée au service du pouvoir dans le sens où l'on peut établir un rapport entre l'idée et la domination (qui) est le propre de toute idéologie (ibid.); ce rapport entre l'idée et la domination sous-tend l'action d'agents à l'œuvre dans la sphère impliquée, chaque action (le fait de "dénoncer" l'un ou d'encenser l'autre...) établissant le plus ou le moins d'un groupe dans son rapport à la domination de ladite sphère.

Se posent ici les problèmes de l'Avant-garde et du rapport qu'entretiennent entre eux les agents et les diverses institutions impliqués. Il s'agit, pour les différentes tendances de la modernité, de faire jouer un discours d'autorité, d'arriver à imposer une "lecture" adéquate du texte moderne, de faire parler un appareil critique qui sorte des lieux de la tradition de façon à sensibiliser de nouveaux lecteurs à la production moderne. Dans ce sens, c'est une pensée au service du "pouvoir-dire», ce pouvoir s'exerçant autant par la fiction que par la critique.

Ces quelques exemples ne suffisent pas à montrer comment la modernité, dans son articulation conceptuelle, est investie par l'idéologique, ni comment elle participe à son système. Nous supposons qu'un "glissement" s'est produit quelque part, et que ce glissement serait l'amorce de «l'élaboration idéologique $"$ du discours sur l'actuel et le contemporain. Un des effets de l'idéologique serait de gommer un lexique déjà contraint. Entre autres, le réseau sémantique mis en place par l'étiquette de la "Nouvelle Écriture» aurait, à l'usage, "migré" vers d'autres lieux où divers agents auraient repris à leur compte les pistes que la N.E. avait amorcées.

Mais comment, du "pouvoir-dire» tracé par la N.F., sommes-nous passés à «l'appellation contrôlée » revendiquée par une École dont le moins que l'on puisse dire, c'est qu'elle se réclame et s'autorise de ce droit de gestion du texte moderne.

\section{Du «thème» à l'idéologie de la modernité}

\section{Un thème: la «Nouvelle Ecriture»}

Nous entendons le thème de la Nouvelle Écriture au sens de Bakhtine, comme un système de signes dynamiques et complexes, qui s'efforce de coller de façon adéquate aux conditions du moment donné de l'évolution ${ }^{6}$. Pour lui, le thème doit aussi pouvoir s'appuyer sur une certaine stabilité de la signification. Or, c'est précisément ce qui fait défaut à la modernité «d'aujourd'hui»: tous les signes dynamiques l'accompagnant sont déstabilisés, saturés par l'emploi.

Les signes dynamiques de la N.E. - le corps, la quotidienneté, etc. s'inscrivaient dans un réseau sémantique et métalinguistique qui la démar- 
quait, ou plutôt la marquait dans la marge. La N.E. rejoignait tout ce qui était considéré comme marginal par l'institution littéraire pour en faire un système - une valeur - où toute "nouvelle écriture" devait forcément s'inscrire. Une contiguïté sémantique entre rupture/marge et nouveauté s'impose comme poncif esthétique: tout ce qui est en marge appelle à la nouveauté et, par contagion de sens, tout ce qui est nouveau sera nécessairement en marge, c'est-à-dire hors de tout cadre institutionnel consacrant les «valeurs sûres» de l'ancien.

La modernité se définissant comme une tradition de rupture - amorcée par la N.E. -, on ne peut que s'étonner, avec Nicole Brossard, que la modernité ait une tradition $!^{7}$ Considérée comme la naissance de la modernité québécoise, la N.E. (tout au moins dans les descriptions qu'on en a données lors du colloque NBJ) se présentait comme une façon d'être, de vivre, et de sentir la vie et l'écriture dans ce qu'elles avaient de plus marginal autant par les thématiques traitées que dans les façons de les mettre en forme.

Le colloque fut donc l'occasion pour la N.E. de poser sa nécessité, son urgence et sa marge, tout en insistant sur celle-ci: se glorifier d'une certaine marginalité... (NBJ, p. 123); nous n'avons fait que relever son besoin le plus intime qui n'est jamais ailleurs que là où ne se trouve pas la tradition (NBJ p. 126). Tout un réseau sémantique se met en place pour définir cette marginalité: l'implicite, le désir, l'indicible, le manque, l'urbanité, la quotidienneté, le corps, etc., servent d'embrayeurs et de renvois directs à la N.E.

Si le thème de la N.E. remplit une fonction, c'est celle d'être métalinguistique et/ou référentielle, celle de créer son propre référent. Les «signes» qu'elle mettra en circulation n'auront pour effet que d'ancrer ce référent, le faisant fonctionner dans l'immédiateté des textes seconds (NBJ p. 165). Dans cette relation autoréférentielle, la Nouvelle Écriture parle le texte dans le texte (NBJ p. 165), et nulle autre étiquette ne viendra s'appliquer que celle que l'on s'accole de l'intérieur. C'est bien d'une "nouvelle" écriture qu'il s'agit, et non d'une banale "écriture" nouvelle: Le substantif faisant sa marque, la nouvelle écriture devient un moteur d'écriture; le mal d'écrire, une caution d'écriture.

S'ajoute aussi au thème de la N.E. le caractère théorique de l'illisibilité (NBJ p. 58), comme mode de mise en déroute de la lecture. Ce sera l'un des pôles centraux de la N.E., jusqu'à ce que le thème glisse vers une "certaine» idée de la modernité. Ce thème s'organise alors en système, imposant (à l'usage) son propre code avec son système de référence «moderne" (mythes contemporains, etc.). Il arrive ainsi à imposer non plus son discours pour «faire moderne», mais son discours sur une modernité pour faire le nouveau.

\section{De l'idéologie dans le thème}

Le texte de la Nouvelle Écriture a-t-il créé un leurre à savoir qu'il a pour fonction d'excéder toute critique et toute théorie? (NBJ p. 165). On pose déjà le problème de la relation de la N.E. avec son discours d'escorte, comme 
pour en illustrer le caractère «illisible». La notion d'illisibilité devient un des points forts de la N.E., dans ce qu'elle vous oblige à ne pas comprendre quand on la regarde (NBJ p. 13). Toute production de sens se posera en "marge" du lisible, lisible étant pris dans sa relation au conceptuel, non pas de la stricte accessibilité de lecture, mais au sens de conceptualisable (par une théorie).

Par ce caractère non-conceptualisable de l'illisibilité, c'est la nécessité (de faire ouvre) qui reprend la "gageure du lisible»: surtout, ne pas théoriser la nécessité, mais la questionner, avant même qu'elle ne s'inscrive, au présent, en tant qu'écriture, nouvelle (NBJ p. 147).

Nous retrouvons de l'idéologie dans le thème, dans la stricte mesure où le thème (la N.E.) distribue ou exacerbe les efforts et les luttes symboliques des groupes culturels qui s'en réclament sur l'horizon d'un marché symbolique. Ces efforts montrent comment on tente d'enraciner le «mode de lecture» de la N.E.: Ce qui différencie la Nouvelle Écriture, c'est le débordement, les excès, la germination d'un autre langage (sic) sans cesse émergeant, les textes explicites qu'elle dit le plus souvent sans le dire. La Nouvelle Écriture (...) pose avec constance d'autres textes (NBJ p. 166).

Tout le réseau sémantique du discours sur la N.E. prend forme et tend à recevoir une valeur sociale, c'est-à-dire tend vers des valeurs socialement échangeables. Des termes comme immédiats, cercle/spirale, indicible, implicite, etc., vont circuler d'abord librement puis, de plus en plus, ils seront tendus par la modernité. D'un symptôme d'une nouvelle écriture qu'ils étaient, ils deviennent la "marque» du texte moderne et vont circuler de moins en moins librement, ancrés qu'ils sont par les valeurs que soutient la modernité. Chaque signe est saturé axiologiquement et, comme le disait l'autre, c'est comme si les mots s'usaient...8.

\section{Des présupposés au service d'un lexique restreint (contraint)}

La "volonté" de faire rupture va aussi engendrer un questionnement sur le discours de la rupture et de son lexique. Sans poser tout de suite la question du contenu de la N.E., il nous faut envisager la relation qu'elle entretient avec le langage et l'image qu'elle donne du travail qui s'y opère.

Car, j'insiste, le texte moderne fonctionne sur un présupposé de dire plus qu'il ne dit. Le texte de la nouvelle écriture fonctionne à l'implicite (NBJ p. 167). La polysémie place le logos, le dire, dans un espace mental rempli des possibilités d'une perspective qui déplace allègrement le sens (NBJ p. 67). On va même jusqu'à accorder aux femmes un "sixième sens" en ce qui regarde le sens de l'écriture/sens de la lecture (NBJ p. 64). C'est, à tout prendre, une résolution vers la mythologie du Dire, de la "révolution de 
l'intérieur» placés sous la «ligne juste» du langage. L'implicite travaille ainsi le texte par la rupture du Cercle à la Spirale, c'est-à-dire en y cumulant du sens déjà là, y ajoutant un sens "indicible» qui donne une valeur "nouvelle» au texte, aux mots.

On présuppose allègrement la langue comme étant piégée, "bourgeoise» et, dès lors, on transpose l'explicite des mots en un discours implicite, neuf, pouvant raccorder le désir de celui ou celle qui parle, à la réalité contemporaine des inavouables (NBJ p. 57). Mais, comme le note A. Marino, voulant saborder le langage, c'est précisément le langage qui finit par saborder l'Avant-garde 9 , ce qui guette aussi notre modernité dans la mesure où la langue peut servir quelque pouvoir que ce soit (au même titre que la science), y compris le pouvoir du langage: ce que les «gens du texte» semblent vouloir dépasser (par la N.E.), voire éviter.

La modernité, avec ses propres termes eux aussi saturés d'idéologie (au sens de Bakhtine), tente de mettre en place un système qui serait exempt d'une éventuelle récupération de quelque côté qu'on la regarde.... C'est oublier que la récupération (du style, du lexique, du genre, etc.) ne vient pas nécessairement toujours de l'extérieur...

\section{La modernité comme moteur d'écriture}

l'écriture de l'écriture reste une voie importante de ce qui retrace le réel, à côté de ce que révèlent la parole des femmes et les textes explorant la trame du vécu ${ }^{10}$.

\section{Modernité ou nouveauté (Forme ou Contenu)?}

Écrire sur l'acte d'écrire devient le moteur d'écriture de la modernité. On met ainsi sur l'avant-scène du texte le prétexte de toute écriture moderne, comme une mise en abyme perpétuelle. Cette conscience de la difficulté d'écrire émerge en même temps que la venue des femmes à l'écriture (tout au moins leur accès à l'appareil éditorial). En effet, plus de la moitié des écrivaines ont fait leur entrée dans le champ littéraire à partir des années soixante-dix et ont, par le fait même, fortement influencé l'écriture. C'est peut-être même l'arrivée des femmes qui a imposé un certain "ton» à la modernité, une certaine façon d'entrevoir la réalité du texte. Le corps, la ville, la quotidienneté ont envahi le discours poétique et y figureront dorénavant l'éclatement (du corps), le mythe (de la ville), et le vécu (la quotidienneté): ainsi, c'est à partir du signifiant femme que le livre s'ouvre sur le monde mettant à nu une pensée essentiellement contemporaine (31.3).

La modernité québécoise fonctionne, comme toute Avant-garde, dans la réification de l'actuel et de la nouveauté. Pour A. Marino, l'Avant-garde littéraire

...cultive et exalte la "nouveauté» spécifique de "l'époque». L'Avant-garde a, au plus haut degré. la conscience d'être contemporaine, synchronisée, intégrée, et de participer à la vie 
immédiate, actuelle. Elle est éminemment "présentéiste», "simultanéiste". (...) Il n'est pas de théoricien de l'Avant-garde qui ne se réclame, d'une manière ou d'une autre, de l'idée de présent, de moderne et d'actualité"1.

Ces thèmes, d'implicites dans la thématique de la Nouvelle Écriture, deviendront explicitement actifs pour la modernité en y faisant jouer une "nouvelle mythologie" du corps, de la ville, du quotidien. L'éclatement du langage se retrouve "rassemblé» autour d'une problématique nucléaire qui s'échappe du Cercle par la Spirale. Cette notion (la Spirale) est d'ailleurs centrale à toute la modernité québécoise: au contraire du Cercle qui se referme sur lui-même (qu'on pense seulement aux Cercles d'écrivains, aux Écoles, etc.). La Spirale se veut apporter quelque chose de neuf aux discours antérieurs, faire oeuvre de reconstruction par rapport aux discours qui "déconstruisent ": le livre est hanté par le désir de nommer ce qui a été déconstruit par les discours antérieurs (31.3). La Spirale "ajoute du sens" là où le Cercle l'enferme.

Plus encore, la Spirale, c'est la déroute, c'est la marge du Cercle. Ce sème sera repris par plusieurs et réinvesti dans leurs pratiques d'écriture autant critiques que créatrices. Le magazine Spirale né d'un désir de renouveler le langage critique... (35.3) entend ajouter, par la Spirale, du discours sur du discours, faire courir le sens de «l'illisibilité» moderne pour lui donner un point d'appui, ancrer les signes dynamiques du texte moderne.

Arcade, une spirale d'écrivantes, reprend l'idée du Cercle éclaté et pose la création en relation directe au dire «ajouté», pris et repris par la connaissance, ajoutant des "connaissances différentes" qui brisent le Cercle. Le Cercle pourrait être considéré comme une figure masculine, la Spirale comme féminine, appelant le "sixième sens" dans l'acception que Nicole Brossard en donnait pour œuvrer dans la modernité.

\section{Modernité et «nouvelle» césure (censure)}

Dans le foisonnement des textes "modernes" circulent plusi:urs approches et pratiques de la modernité. Plusieurs avenues thématiques ou formelles ne se rejoignent, souvent, que par le fil de plus en plus ténu de la nouveauté en écriture. Parler du corps, parler du texte dans le texte, dire le corps/texte, la ville et l'urbanité, "formaliser» des thématiques «nouvelles" paraît en appeler de la modernité (par la forme tout au moins), mais tous ne sont pas appelés par elle, tous ne suivent pas sa voie, ni l'idée que la «critique moderne" se fait d'elle... et des contenus qui peuvent y circuler.

La modernité, on l'a dit, repose sur une équivoque, à savoir qu'elle tend à vouloir voir le texte moderne comme un Tout Synthétique. Chaque partie de ce Tout (de textes, d'œuvres ou de titres) accumulant le sens déjà là, élaborant l'idée même de modernité. On la présente presque comme un mouvement social. 
Pour certains critiques de la modernité à Spirale, il semble de plus en plus évident que la modernité se construit sur un amalgame, et que le "Tout Moderne" ne peut que refléter des modernités; d'où des effets de ruptures qui se font sentir de l'intérieur. Fondée sur la rupture (entre, principalement, anciens et modernes), la modernité a du mal à conjuguer la rupture que provoquent "certaines modernités».

Dans le discours critique sur la modernité que tiennent les chroniques de Spirale, on note qu'une distance s'établit entre "l'objet moderne" qu'est le texte, et le "sujet moderne » qui s'investit dans "d'autres lieux de déroute" (au sens littéral du terme où il déroute la lecture). L'indice de Dieu sera, dans Spirale, un pôle de tension: "forme moderne" et "contenus archaïques" mettront en relief des enjeux idéologiques jusque-là passés inaperçus.

Au fil des numéros de Spirale apparaissent donc une nouvelle terminologie et l'utilisation très modalisée de certains concepts. Les parenthèses et les guillemets abondent et témoignent d'un malaise à propos de ces thématiques que l'on juge dépassées ou carrément rétrogrades. Le malaise se renforce du fait que ces textes sont "absolument modernes"!

Dans les énoncés méliorés, par exemple, on réifie la "chose littéraire». Il n'y a plus de sujets parlants: "ça» parle, "ça» agit, "ça » produit le sens. Ce "ça» est un "être" idéologique (Angenot), déterminé et défini uniquement par l'ensemble des maximes isotopes où le système idéologique leur permet de prendre place ${ }^{12}$.

Dans les énoncés péjorés cependant, ce n'est plus le «ça» qui agit, mais le texte, l'auteur. Il s'agit d'un tout autre ordre de représentation: on glisse du «ça " parle moderne au «il " parle traditionnel. Le "ça » synthétise la modernité du texte et en fait un "sujet» organiquement lié à la problématique de l'écriture. Un texte moderne est un texte qui assume le "ça» de l'écriture (au sens psychanalytique du terme).

Apparaît ici le problème des contenus où "ça parle» de façon tout aussi moderne, mais où "ça parle" des indicibles et des éclatements non valorisés par la modernité, où ça fait une brèche dans le procès moderne de la textualité. L'indice de Dieu est l'exemple le plus frappant de la contradiction entre modernité formelle et modernité contenuiste. Le regard critique est bien obligé de reconnaître le côté moderne du "ça parle» à relent religieux, mais on le fait tout en mettant en garde contre ce qui pourrait signaler des voies d'écriture (34.8). C'est seulement à partir de l'indice de Dieu et de son discours que se posera le problème des modernités progressistes (!) et archaïques (!). Le discours critique sur la modernité s'est installé dans un système de références à partir duquel il «juge» les productions actuelles (à remarquer que proférer un jugement et énoncer une critique ne participent pas du même procès). Une grille s'est imposée là où l'on voulait échapper à l'analyse, voire la dérouter (et dérouter l'analyste).

Le texte moderne québécois réside aussi ailleurs que dans les lieux mentionnés (H.R. et NBJ). Ces lieux sont cependant marginalisés par la critique. 
Une raison peut-être: il y a des lieux de publication "modernes", il y a des lieux de publication "anciens", mais il y a aussi des lieux de publication "ambivalents», où l'on semble à la fois produire anciens et modernes. C'est le cas du Noroît. On y produit des poésies très diversifiées, et c'est explicitement la politique de la maison.

Tout en notant l'importance de ce lieu d'édition pour la poésie québécoise, on lui reproche son «absence d'École». C'est un lieu de modernité inclassable: mais si le Noroît est un véhicule important pour la poésie québécoise, il ne répond malheureusement pas aux attentes. Toutes les poésies s'y côtoient... (37.8) (c'est nous qui soulignons). Si le Norô̂t ne répond pas "aux attentes", celles-ci restent cependant dans l'explicite le plus absolu et le cela-va-de-soi le plus évident: la modernité a des lieux de production spécifiques, le traditionnel aussi; ne mélangeons pas les genres: ce que produit le Norô̂t reste inégal. Cette attitude est regrettable pour le renom de la maison et l'on souhaiterait une politique plus cohérente (37.8).

On a affaire ici à un jeu de place. Comme le note Bourdieu, la raison d'être d'un discours ne réside jamais complètement dans la compétence proprement linguistique du locuteur; elle réside dans le lieu socialement défini à partir duquel il est proféré... ${ }^{13}$.

Le genre de critique adressée au Noroît ne vise en fait qu'à circonscrire les limites du champ où circulent les biens symboliques; aussi, dans les limites de ce champ, aura-t-on tendance à dévaluer les autres usages possibles, mais non conformes aux normes du champ ${ }^{14}$. Cette chronique est donc une condamnation implicite de la transgression de la "marge": les modernes publient avec les modernes, les anciens avec les anciens! L'éclatement sur papier est une chose que l'on semble ne pas pouvoir tolérer dans la pratique. Cette critique agit comme une mise en garde: attention aux cadres de la marge!

\section{L'implicite d'une politique éditoriale (d'un présupposé moderne à une modernité supposée)}

La modernité fonctionne sur la base de présupposés : une implicite complicité du texte et de la lecture, une relation ludique entre le texte et le «sujet parlant ", une écriture qui s'écrit, etc. Tout est beau et tout va bien jusqu'au jour où commencent à émerger des questions au "ça parle». Bien sûr que "ça parle" (on est bien obligé de concéder, maîtrise de la langue oblige!), mais "ça parle" à qui et de quoi? C'est dans cette direction que semble s'orienter la polémique et, pour l'une des rares fois depuis la disparition des revues spécialisées comme Champs d'application, Chroniques et Stratégies, entre autres, il sera question de contenu à propos d'œuvres formellement modernes.

11 n'est pas tout à fait étonnant non plus que cette polémique autour de "contenus" s'amorce autour de personnes ayant déjà travaillé à ces revues. La publication dans Spirale d'une chronique à propos d'une exposition (voir plus spécifiquement le numéro 35) amènera la démission de trois personnes 
(sur six) du comité de rédaction. Leur texte de démission dénonce fortement l'indice de Dieu là où il parle, c'est-à-dire à travers les référents modernes de l'éclatement, du Cercle spiralé et de l'indicible (notons en passant que l'indicible, c'est aussi «Celui qui n'a pas de nom», le Dieu innommable des Juifs de l'Ancien Testament).

Comment, disent-ils, ne pas voir que l'écriture qui s'y joue, pour érudite qu'elle puisse être, pour moderne qu'elle se prétende encore, dérape singulièrement du côté des Écritures (35.3). Dans le recensement que nous avons fait, c'est le seul article qui relie le «jeu de l'écriture" à une problématique de contenu, comme si tous les autres textes critiqués, pour modernes qu'ils sont, se situaient aussi dans la marge du soupçon (!). Pour les autres, «horsmodernité", le problème ne se pose même pas : ils sont tout bêtement "naïfs" .

Pour moderne qu'elle se prétende encore place ce texte dans le sillon de la modernité tout en le démarquant de l'idée. Ávant cette brèche, le texte est "moderne" par sa recherche formelle, par l'inscription du corps, etc., et on n'y relie le contenu que par le propos, c'est-à-dire par sa "formalisation». C'est ici l'inverse qui se produit: peu importe la forme qui l'habille, le «contenu christique" est expédié hors de la modernité: constatons-le: ce texte est sexué et le corps célébré, derrière un écritoire en linceul, s'y exhibe mâle sans concession, narcissique et souffrant... (35.3). Le texte sexué, le corps célébré participent ailleurs à l'idée de modernité supposée du texte qui fait ici problème, et qui incite à une vigilance élémentaire (34.8).

En fait, les démissionnaires s'élèvent contre la "récupération" ou l'utilisation des "formes modernes" à toutes les sauces, puisqu'ils entérinent toujours leur soutien à la modernité. Ils continuent d'ailleurs de publier à Spirale et leur démission (du comité de rédaction) n'est pas une rupture mais une pétition de principe, un cri d'alarme. L'acte relève plus du pamphlet que de la polémique: une mise en garde contre une politique éditoriale "pluraliste à tout prix». On semble ne plus pouvoir énoncer sans dénoncer...

La réplique de l'auteur de la chronique controversée mériterait une analyse en soi tellement elle met en jeu une théorie de la "réplique» et de l'énonciation. Dans ce sens cette réplique participe de la polémique par ce qu'elle peut avoir de "valeur distinctive»: c'est-à-dire qu'elle entre dans une relation objective de concurrence par rapport à d'autres produits symboliques, d'où découle un profit de distinction en rapport à un univers hiérarchisé d'écarts à une forme de discours reconnue comme légitime ${ }^{15}$.

Au-delà de la prise de bec, il y a des énoncés qui dénotent le «projet unificateur" de la modernité: la modernité apparaît, même maintenant, morcelée. C'est, entre autres, le but de Spirale, de montrer qu'il ne règne pas d'unanimité dans les productions québécoises de la modernité, que l'unité n'était peut-être qu'un effet de surface, mais qui a sans aucun doute été utile historiquement (35.3: "L'aire du soupçon», réplique aux démissionnaires).

L'implicite de la modernité (le Tout Synthétique) tend à se redéfinir en fonction de la modernité supposée de certains lieux de publication. Un lieu 
dit de modernité suppose qu'on attend de la production de ce lieu de la modernité, et tend à relativiser «l'écart» qui s'y pratique par la publication de textes "archaïques». Le contenu de toute modernité n'est plus au-dessus de tout soupçon et la redéfinition, tout au moins dans son articulation «théorique", devra relever de l'ensemble esthétique de l'œuvre moderne. C'est-àdire qu'on sera attentif dorénavant $\dot{a}$ une certaine forme d'obsession archaïque qui refait surface de façon surprenante dans un lieu dit de modernité (34.8).

L'idéologie de la modernité fonctionne selon un système d'opposition, avec ses pôles négatifs et positifs: plus moderne/moins ancien, plus ancien/ moins moderne, et se joint à la pensée partisane, collective, dissimulatrice, rationnelle (dans le plus et le moins que nous avons indiqué); elle participe ainsi à l'idée de pouvoir dans ses luttes pour la domination de champs culturels spécifiques.

Cependant, quand la "Spirale» a peine à déborder le "Cercle», dáns le fait précis de son non-dit, la modernité se voit refoulée à son point d'origine quand son non-dit devient un «non-disable». Elle ne peut que retourner à son point de fuite:

Se glorifier d'une certaine marginalité, c'est avant tout se définir un certain territoire pour mieux s'y installer. Il ne suffirait pas. pour saisir le marginal dans l'écriture de pointe, d'en préciser certains contenus (...) car même cela fait, on en aurait pas terminé avec sa fuite, sa cause, son biais (NBJ p. 123).

\section{(c'est nous qui soulignons)}

C'est bien avec la "pureté originelle» (de la N.E.) qu'une certaine modernité fait défaut, là où le convenu disparaît au profit d'une différence (NBJ p. 123).

\section{Une production restreinte}

\section{l'intertextualité comme gage moderne}

(la citation de ses "pairs")

Des noms aussi et des épitaphes et des dédicaces pour habiter le livre, pour l'accompagner. Il n'écrit pas seul. (38.5)

Il faut se méfier de ces livres qui donnent, à chaque texte, au moins un vers «à citer», voilà trop souvent le leurre du lisible, la catalyse du sens, ce qui assure, cristallise la lecture jusque-là déployée, éclatée.(31.16)

Voilà deux conceptions fort différentes de l'idée que l'on peut se faire de l'usage de la citation! Tantôt une initiation mal à propos, tantôt une «lecture» qui ancre l'inter-texte. La citation sert toujours la sororité et la paternité, le désir de poser avec constance d'autres textes (NBJ p. 166); cela revient à ancrer le système de référence, l'ordre de la représentation, dans ce qu'ils servent à persuader ceux qui pensent comme vous (NBJ p. 125). 
Bourdieu nomme ce procédé le langage d'autorité, dans ce qu'il doit contribuer à sa propre crédibilité. La citation sert ici de raccourci et son usage social tend à signifier: oui, je pars d'ici, j'ai fait telle lecture, tel auteur (toujours contemporain) est génial, et moi, je reconnais ce génie!... Autrement dit, reconnais-moi et je te reconnaîtrai...

Ce jeu de l'inter-citation renvoie directement à la Spirale comme concept de cumulation de sens et de connaissances. La citation part de là où on en était (l'état de la science, pourrait-on dire!), pour marquer la distance parcourue par le "nouveau texte». C'est aussi un système de référence spatiotemporel servant à légitimer le sens et l'importance des textes précédents, ceux de ses pairs.

C'est l'École, le Groupe qui se fait lui-même la place qu'il veut occuper dans le champ de production restreint du poétique, et rien ne semble plus efficace que de se reconnaître soi-même pour contrer la non-reconnaissance institutionnelle.

\section{Un nouveau lecteur: forme et formation}

Si ceux qui veulent défendre un capital menacé (...) sont condamnés à une lutte totale (...) c'est qu'on ne peut sauver la compétence qu'à condition de sauver le marché, c'est-à-dire l'ensemble des conditions sociales de production et de reproduction des producteurs et des consommateurs ${ }^{16}$.

Cette note de Bourdieu situe bien le fonctionnement de l'Avant-garde. La production restreinte force peut-être ce jeu d'auto-référence et d'autoreconnaissance. L'émergence d'une "nouvelle écriture» reposait sur la formation de "nouveaux lecteurs", de façon à pouvoir ancrer quelque part ce "lieu de déroute», lui donner lecture, des routes à suivre. Le problème que pose la réception trouvera sa solution à travers l'institution même dont on se veut la marge : les maisons d'enseignement. La plupart des auteurs et auteures de la modernité enseignent en effet au niveau collégial (quelques-uns à l'université). C'est prendre au pied de la lettre la "nouvelle écriture" dans ce qu'elle appelait nécessairement de nouveaux lecteurs. Qu'à cela ne tienne, nous les formerons:

La place qu'on lui donne (à la N.E.) dans l'enseignement collégial, sur ce point de vue en avance sur l'enseignement universitaire, affirme davantage encore cette vivacité de création (NBJ p. 115).

En plus de permettre la "survie économique» de certains lieux de publication (certains titres sont littéralement des best-sellers de programme), l'auto-formation favorise l'appropriation des "codes de lecture" du texte moderne et en assure la continuité (sa reproduction). La réédition de certains textes, hormis l'importance intrinsèque qu'ils peuvent avoir, oriente la modernité vers "l'institutionnalisation collégiale" par opposition à celle de l'université, que l'on veut à tout prix éviter puisque vous y dormez dans l'articulation théorique (NBJ p. 17). La pratique du discours sur la modernité 
s'inscrit aussi dans cette lutte pour une recherche et un discours différent qui veut marquer sa différance (au sens de Derrida) par rapport au discours universitaire.

L'idée de modernité, on l'a vu, participe au procès idéologique par sa fonction comme par sa nature. Tout discours implique des enjeux idéologiques et politiques, et celui sur la modernité n'y échappe pas malgré certaines prétentions: l'énonciation a horreur du vide idéologique. Comment peut-on s'opposer aux idéologies constituées sans en reprendre le même procès? Un discours se voulant novateur peut-il innover jusqu'à la limite de sa fonction, c'est-à-dire innover aussi dans sa fonction narrative et idéologique?...

1. Le terme est de Madeleine Gagnon.

2. Nous n'avons pas voulu personnifier ce débat où déjà trop de «noms" circulent. Aussi, chaque citation ne sera suivie que par sa référence matérielle; le lecteur pourra, s'il le juge pertinent, retracer facilement chacun des auteur/es. Les renvois sont donc indiqués comme suit:

- (NBJ, p. ...) renvoie aux actes du colloque sur la Nouvelle Écriture de la Nouvelle Barre du Jour, nos 90-91, 1980;

- (34.x) renvoie au magazine Spirale de l'année de publication 1983: 34 = le numéro, $x=$ la page.

3. O. Reboul, Langage et Idéologie, Paris, PUF, 1980.

4. Pour des raisons d'économie, nous avons limité notre appareillage conceptuel à un seul auteur, ce qui n'implique pas que d'autres approches ne soient pas souhaitables pour aborder l'Avant-garde et son discours. L'aspect opératoire de l'approche de Reboul, délimitant l'idéologie en cinq points, nous a paru cependant plus "rentable» dans le cadre de cet article.

5. O. Reboul, op. cit.. Les cinq points auxquels nous nous référons sont explicités aux pages $2 \mathrm{l}-25$.

6. M. Bakhtine. le Marxisme et la philosophie du langage, Paris, éd. Minuit, 1977. p. 143.

7. N. Brossard, in Vouloir la fiction. NBJ no 141, p. 85.

8. C. Beausoleil, ibid. p. 64.

9. A. Marino, «Essai de définition de l' "Avant-garde», revue de l'Université de Bruxelles, 1975. p. 64-120.

10. J. Royer, «La fin de l'illisibilité». Le Devoir, 19 mars 1983.

11. A. Marino, op. cit.

12. M. Angenot, la Parole pamphlétaire. Paris. Payot, 1982.

13. P. Bourdieu, "L'èconomie des échanges linguistiques", in Langue française no 34, mai 1977.

14. P. Bourdieu, ibid.

15. P. Bourdieu, op. cit. p. 24.

16. P. Bourdieu, op. cit. 